\title{
ALTERED NEURO STATES
}

\section{Tom Fox}

Today some artists appear driven by an ambition, on the one hand, to inhabit a place of total affect and, on the other, to be drained of affect altogether; on the one hand, to possess the obscene vitality of the wound and, on the other, to occupy the radical nihility of the corpse. Pure affect, no affect: It Hurts, I Can't Feel Anything.

Hal Foster, "Trauma Culture"|

I am a Dunedin-based artist, Father and Poppa; in 2019 I graduated from the Dunedin School of Art, where I completed a Master of Fine Arts degree with distinction. "Altered Neuro States" was my Master of Fine Arts degree exhibition, a collection of 19 large $(2.1 \times 1.4 \mathrm{~m})$ drawings, each drawing built up using finger-prints. The final rendered panels are each windows into my everyday experiences and my struggle to understanding my own personal reality. I'm autistic - I received a diagnosis of Autism Spectrum Disorder level one (ASD), which was once known as Asperger's, when I was 37. As a label it was the most freeing I have ever received. It gave me an opportunity to understand myself and my life in a framework that gave deep insights into my experiences and why I experience them.

As I researched the neurological aspects of autism I discovered that my sensory world is unique, that what I experience daily as being visually overloaded, sensitive to sound and often overwhelmed by them both, had a name, Sensory Processing Disorder (SPD). One problem I have when it comes to processing experiences is that I can't visualise them, I can't recreate them in my mind. I have mind blindness - it is called aphantasia, the inability to create mental images. What I see when I close my eyes are swirling and pulsating dots of light called phosphenes; sometimes I see flashes of fractured images, but they are random and often have no connection to what I'm thinking about.

I think in narratives and concepts; thinking like this is what l've always known. Although it isn't always helpful for navigating the visual overload and distortions that affect me, it is a big part of why I draw them. I want to understand them and thereby understand myself in a more complete way. In this context, my art practice operates as a cathartic process of self-understanding, building bridges of understanding between my experienced reality and my understanding of what those experiences actually are.

For me, the journey of autism has been a journey of self-discovery and an attempt to understand my perceived reality. I study aspects of neuroscience and psychology to use as a framework for navigating my experiences, and my drawing acts as a physical manifestation of my sensory overload. When these things are considered together, I can process and analyse experience in a meaningful way.

In the end, to try and understand perceived reality is a foolish errand as there is no finish line, no satisfying answers and no end of new questions - but like the Fool of the Tarot one must walk the path to become something else.To find any cohesive idea of reality or perceived reality one must look at the sensory system. The sensory system acts as a bridge between the physical world and the internal world. 
The human body receives stimuli through its nervous system which includes visual and auditory information, touch, taste and smell. These stimuli are responded to through the autonomic (which controls subconscious body functions) and somatic (controls both voluntary and involuntary muscle control) nervous systems. Higher ordered or conscious responses are actioned through the prefrontal cortex, which evaluates these automated responses and generates changes that are sent back to the limbic system. ${ }^{2}$ In simple terms, the sensory system is a feedback loop of stimuli, reaction/action, evaluation and adjustment.

According to Harvard psychologist Jeffrey Statinover and neurophysiologist, geneticist and physicist Joe Dispenza, the human brain processes an estimated 400 billion bits of information per second. ${ }^{3}$ The brain acts as a filter of information; everything seen, heard, tasted, touched or smelt is an interpretation of the information the brain is receiving. The interpretations perfomed by the brain are often mistaken as accurate representations of the real world. The human brain is constantly editing and making choices about which information to reveal. In neurology this process is known as sensory gating. Sensory gating is the ability of the brain to manage sensory input. As the brain receives a constant stream of information, the gating function can respond to any changes within the stream, including limiting the information or even stopping it. ${ }^{4}$

Given the estimated 400 billion bits per second of information that the brain is processing, if the brain was not limiting this data then the individual would be completely overwhelmed by sensory information, as happens in ASD and SPD. One example of sensory gating is the phenomenon known as the cocktail party effect ${ }^{5}$ - the process of filtering out background sound while someone is focused on a conversation in a noisy environment, as in a cocktail party. The brain accesses the auditory inputs and segregates them into streams, and then chooses which stream of information to 'dial up or down.'This allows people to hear the conversation rather than a wall of incoherent noise. Without a sensory gating function this would be impossible. ${ }^{6}$

Another way to look at sensory information and sensory awareness is in terms of high resolution and low resolution, just like jpeg images. If the 400 billion bits of information per second is viewed as a high-resolution image, and the sensory information that the conscious mind is aware of as a low-resolution image, then this can be seen as a map to understand how the human sensory system works. The brain is efficient at giving just enough information to navigate in the real world, offering low-resolution information from which to make quick judgements and decisions. The way low-resolution information works for navigation can be compared to an internet business like Amazon or eBay. When the user scrolls products on their websites, the images of the products are displayed at low resolution, which uses less computing resources. It keeps the products in front of the viewer while scrolling through items and the system stays in an optimal state as resources are not wasted on rendering high-resolution images.

Being hypersensitive to sensory information like light, smell or sound means that a person living with a sensory disorder must constantly process high-resolution information. This is often overwhelming; however, it also means that the individual may have more 'potential' information available to them from which to draw creative responses. High-resolution information needs to be processed in order to navigate the world, and that process can overwhelm the brain because of the level of sensory intensity that needs to be managed. Yet the process of trying to navigate high-resolution information can itself lead to novel connections that would otherwise be unavailable or far harder to make at low resolution.

Perceived reality is simply a result of how the brain processes sensory information. As humans, we haven't evolved to have a sensory system that reflects the absolute truth of the surrounding world, but rather one calibrated in terms of survivability. Being a successful life form means that we can survive the environments we inhabit and are able to reproduce offspring that can do the same. One example of the limits of the human sensory system is the human eye. Because the eye can only see $0.0035 \%$ of the electromagnetic spectrum, ${ }^{7}$ we only see a miniscule amount of the potential perceivable world. Other life forms perceive a differing range of the spectrum and experience the world differently to humans - for example, larger ranges of colours or ultraviolet patterns on flowers. Ultimately, evolutionary success does not necessarily require sensory truth. 


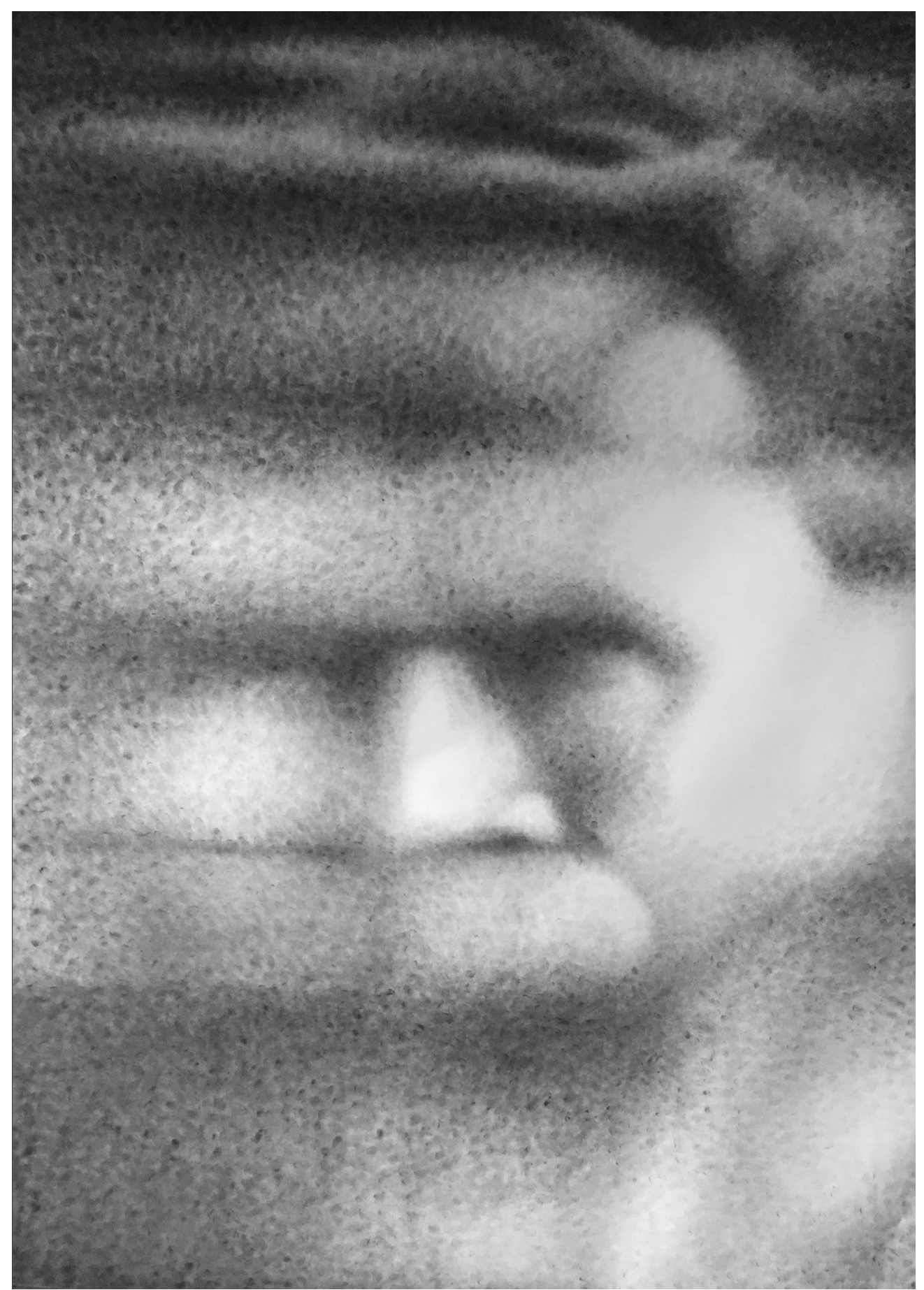

Figure I.Tom Fox, Untitled, 20 I 8, ground chalk pastel on paper, $208 \times 146 \mathrm{~cm}$. 


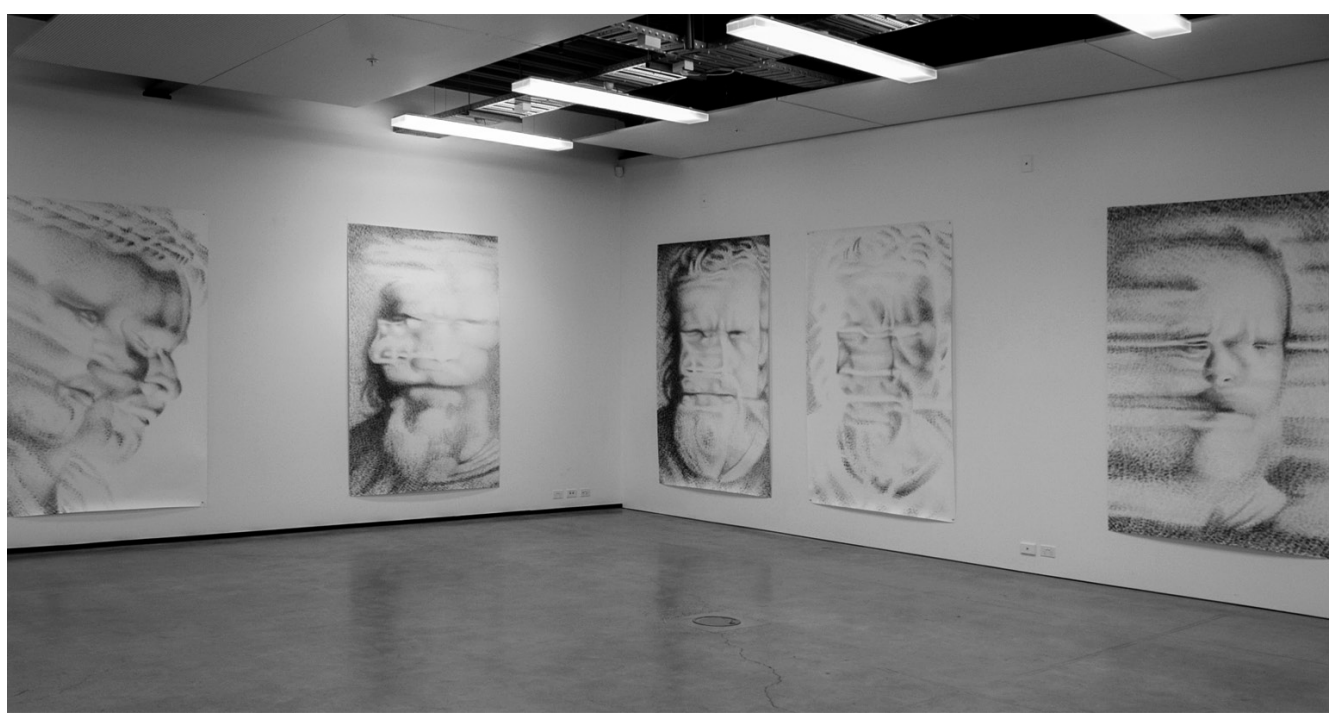

Figure 2.Tom Fox, "Altered Neuro States," 23-26 September 2019, installation view. Dunedin School of Art Gallery.

On top of this, people with ASD and SPD often experience regional brain changes, ${ }^{8}$ including but not limited to changes of volume in the hippocampus and amygdala and changes to the brain's white tissue micro-structure. ${ }^{9}$ Any changes to the brain regions affect brain function, which can then alter how the sensory system works and how a person experiences sensory information. In a nutshell, brain changes affect the sensory system and experienced reality.

By journeying down the rabbit hole that is the human brain, I hit a major wall of understanding (one among many). If perceived reality is simply the product of sensory information and the ways in which the brain interprets it, then perceived reality is simply my consciousness experiencing my sensory system and therefore can't be representative of a true physical reality. So how does consciousness relate to the physical world? It is at this point that the science of neurology fails and the only way forward is to enter the realm of aesthetics and philosophy.

There are two main systems of thought that attempt to explain reality. The first is physicalism. In nearly all the 'hard' sciences like physics, biology and chemistry, and the 'soft' sciences like psychology, sociology and psychiatry, a system of thought known as materialism or physicalism permeates these various disciplines. While the two names are mostly interchangeable, physicalism is often preferred when discussing metaphysics. I'll use physicalism from this point on: the idea that anything that is experienced or observed must have a cause in the physical world ${ }^{10}-$ a chain of cause and effect.

Applying the notion of physicalism to what we know about brain changes and how they affect sensory processing, the experience of sensory distortions can be directly traced back to brain changes. To be clear, according to the majority view within science my distorted sensory experiences can only be explained by the physical nature and structure of my brain and how that changes the way my brain functions.

This means that the brain and the physical matter it is made from dictates how I (and everyone else) experience reality and, taking the argument another step, physical matter is the source of consciousness. In philosophical language, this makes physical matter the ontological 'primitive' basis of reality - primitive meaning that it can't be reduced further, and ontological referring to the nature of being. All theories of nature must offer one ontological 'primitive', 
and almost all theories with a physicalist basis state that the reality we perceive is grounded in matter; in physicalism matter is often reduced to string theory, $\mathrm{M}$ theory and quantum field theory, among others. I won't explain the details of these theories, but they all build on the same initial observations, then apply differing interpretations and mathematical explanations of these observations. These theories basically constitute mathematical philosophy; while each offers insightful and clever interpretations of observational data collected over a very long time, so far they have proved to be incredibly difficult to test. For now, the theory one might chose (or reject) simply comes down to the aesthetics of the explanation. One major problem arises in the physicalist world view - known in neuroscience and philosophy as the hard problem of consciousness."

The hard problem of consciousness is the point at which physicalism fails, at least for now. Current explanations of consciousness posit that it is a product of some physical structure or combinations of structures of the brain. However, it is yet to be discovered how consciousness has not simply been reduced to the physical nature of the brain - the most we know is that there is a relationship between the brain and mind. In fact, there is currently no way of explaining how the physical properties of matter - like particle spin, mass or charge -can create experiences like feelings of love or disappointment, or the sensation of a stomach ache. This in a nutshell is the hard problem of consciousness.

One competing theory for explaining what we perceive as reality is idealism, ${ }^{12}$ which views consciousness as the ontological primitive, seeing consciousness as a force like the strong and weak nuclear forces or gravity. Idealism holds that instead of strings or membranes, a universal consciousness vibrates and creates the matter we perceive. Although it sounds like new age spiritualism, this theory is actually well supported by scientific observations equally as well supported as the physicalists models, as it uses the same initial observations as physicalism, but applies a different philosophical basis.

According to Stanford physics professor Andrei Linde, a leading exponent of idealism:

Let us remember that our knowledge of the world begins not with matter but with perceptions. I know for sure that my pain exists, my "green" exists and my "sweet" exists ... everything else is a theory. Later we find out that our perceptions obey some laws, which can be most conveniently formulated if we assume that there is some underlying reality beyond our perceptions. This model of [the] material world obeying laws of physics is so successful that soon we forget about our starting point and say that matter is the only reality, and perceptions are only helpful for its description. This assumption is almost as natural (and maybe as false) as our previous assumption that space is only a mathematical tool for the description of matter. ${ }^{13}$

This quote is a reminder that perception is both the starting point but also the end point of the knowable world; everything relies on perception, even science. Everything that can be tested or recorded relies on an observer to record it, and we know from quantum physics that observation can affect the outcome of tests. ${ }^{14}$ The thought experiment "If a tree falls in a forest and no one is around to hear it, does it make a sound?" is an interesting example of observer bias, because according to accumulating observations in quantum physics, the answer is likely to be "no"15 - if there is no eardrum to receive the sonic wave and no brain to interpret it as sound, then there is no sound, only a wave function.

In an essay, "The Mental Universe,"'16 published in the journal Nature in 2005, Richard Conn Henry, professor of Physics and Astronomy at Johns Hopkins University, uses the double slit experiment and other physics and quantum physics observations to argue for the mental nature of reality. He reaches two major conclusions: a) observation necessarily boils down to perceptual experience; and b) the physical properties of the world exist only insofar as they are perceptually experienced.

The truth is that although no one knows what reality is, I need to understand mine to the best degree I can. I am driven forward by that idea. I need to understand my world, even though there are no definitive answers. I need to find meaning in the chaos, and drawing is the tool I use to experiment with the boundaries of my reality. 
Each drawing I make is an exorcism, an exorcism of internal distortions delivered by my sensory system. It takes something material from myself to turn my internal experiences into something concrete - in this case, my fingerprint that carries my DNA and is evidence of my physical reality. Slowly I materialise a sensation, a physical response to a trick of my neurology. Once it is materialised, it can be analysed as a concrete artefact of an abstract experience, a form of communication, something to learn from. My drawings are external manifestations of my sensory world yet they are only a partial truth, a low-resolution physical image of high-resolution sensory information.

Like painting in pixels, print by print, mark by mark, I'm creating a structure from the abstract; every image made is a study of an abstract experience that can then be used to help navigate and understand that experience. While it communicates with me, it has potential to communicate to others a partial snapshot of the chaos of sensory abstraction that I experience.

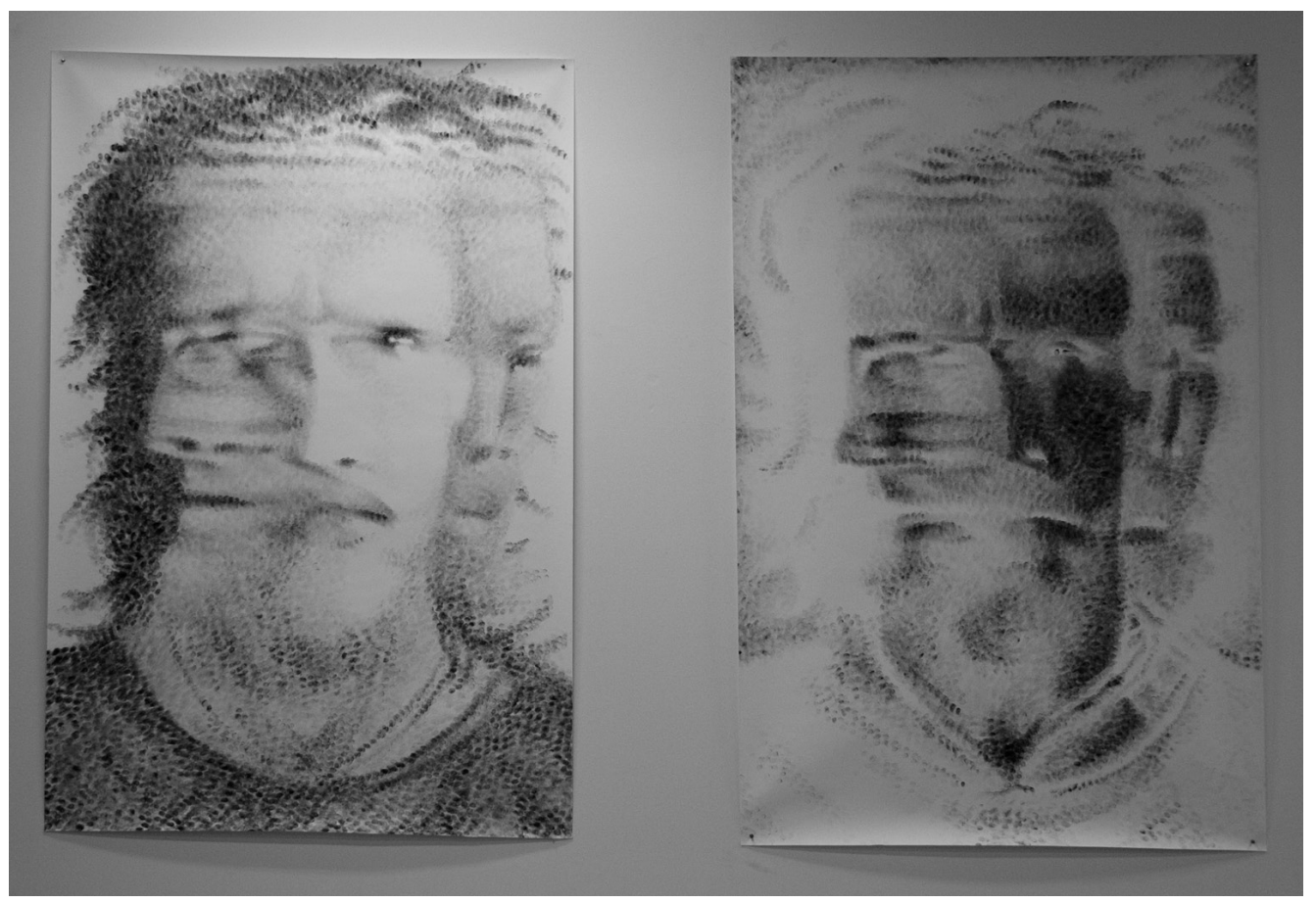

Figure 3. Tom Fox, Differentiation No. 2, 2019,

ground chalk pastel on paper, approx. $201 \times 145 \mathrm{~cm}$ each. 


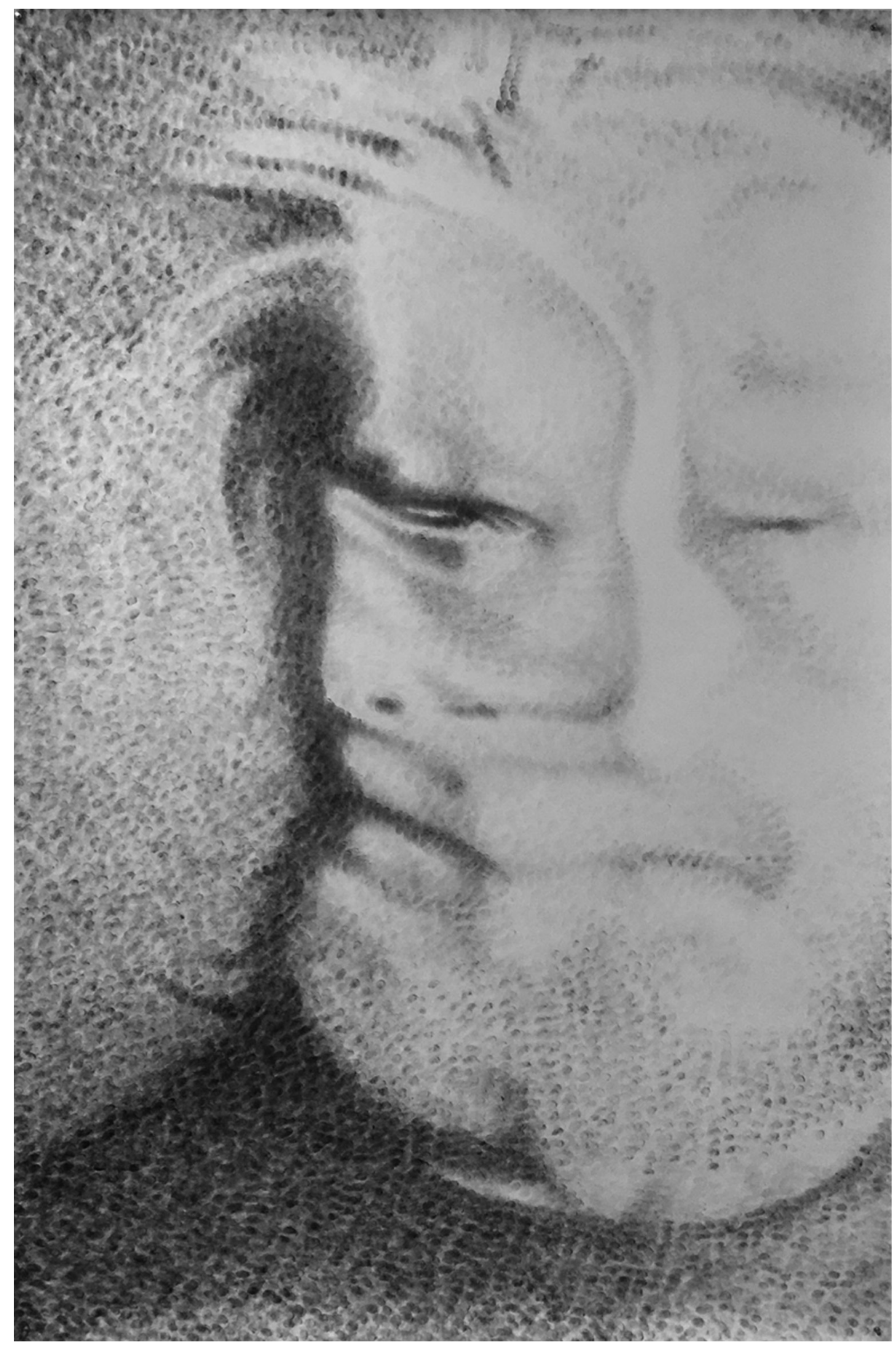

Figure 4.Tom Fox, Differentiation No. 7, 2019, ground chalk pastel on paper, approx. $201 \times 145 \mathrm{~cm}$. 
Tom Fox is a Dunedin based artist, a graduate of the Dunedin School of Art, he works in a variety of mediums including photography, painting and large-scale chalk pastel and paper works. His art practice investigates the effect that trauma and sensory conditions have on an individual's perception of the world.

I Hal Foster, “Trauma Culture," artnet, last modified 7 December 2015, http://www.artnet.com/magazine_pre2000/features/ foster/foster7-26-96.asp.

2 Sonia Martínez-Sanchis, "Neurobiological Foundations of Multisensory Integration in People with Autism Spectrum Disorders: The Role of the Medial Prefrontal Cortex," Frontiers in Human Neuroscience, 8 (2014), 970, https://www.frontiersin.org/ articles/l0.3389/fnhum.20|4.00970/full.

3 Maicon Santiago, "How Much Information Processing the Brain?," Ciencia em novo tempo, last modified 22 November 201 I, https://cienciaemnovotempo.wordpress.com/categorias/fisica-neurociencia/how-much-information-processing-the-brain/.

4 Nashaat N Boutrosa and Aysenil Belgera, "Midlatency Evoked Potentials Attenuation and Augmentation Reflect Different Aspects of Sensory Gating," Biological Psychiatry, $45: 7$ (1999), 917-22.

5 Andrew RA Conway, Nelson Cowan and Michael F Bunting, "The Cocktail Party Phenomenon Revisited:The Importance of Working Memory Capacity," Psychonomic Bulletin and Review, 8:2 (200I), 33 I-5.

6 Ibid.

7 Ava Andrus, “The ColorWe See," Prezi, last modified I4 December 20 I4, https://prezi.com/jqf-we9hkfdw/the-color-we-see/.

8 Gráinne M McAlonan, Vinci Cheung, Charlton Cheung, John Suckling, Grace Y Lam, KS Tai, LYip, Declan GM Murphy and Siew E Chua, "Mapping the Brain in Autism. A Voxel-Based MRI Study of Volumetric Differences and Intercorrelations in Autism," Brain, 128:2 (2005), 268-76, https://doi.org/10.1093/brain/awh332.

9 Y-S Chang, M Gratiot, JP Owen, A Brandes-Aitken, SS Desai, SS Hill, AB Arnett, J Harris, EJ Marco and P Mukherjee, "White Matter Microstructure is Associated with Auditory and Tactile Processing in Children with and without Sensory Processing Disorder," Frontiers in Neuroanatomy, 9 (2016), 169, https://www.frontiersin.org/articles/ I 0.3389/fnana.20 I 5.00 I 69/full.

10 Andrew Melnyk, "Physicalism," Oxford Bibliographies, 20 I5, last modified 25 April 20 I 5, https://www.oxfordbibliographies.com/ view/document/obo-9780195396577/obo-9780195396577-0267.xml.

I I Bernardo Kastrup, "Consciousness Cannot Have Evolved," iai News, 2020, last modified 5 February 2020, https://iai.tv/articles/ consciousness-cannot-have-evolved-auid-I 302.

12 Bernardo Kastrup, "On Why Idealism is Superior to Physicalism and Micropsychism," Metaphysical Speculations, 20I6, last modified 16 January 2016, https://www.bernardokastrup.com/2016/0 I/on-why-idealism-is-superior-to.html.

13 Andrei Linde, Universe, Life, Consciousness, 2015, https://dbx6c2burld74.cloudfront.net/article/I5525 I 7594-78f8303efbd5e37 4b6b50b la4599dcaa.pdf (accessed 12 April 2020).

14 Joshua Kincaid, Kyle McLelland and Michael Zwolak, "Measurement-induced Decoherence and Information in Double-slit Interference," American Journal of Physics, $84: 7$ (20 I 6), 522-30, https://doi.org/ I 0.1 I I 9/1.4943585.

15 Jim Baggott, "Quantum Theory: If a Tree Falls in the Forest ...," OUPblog, 20 I , last modified I4 February 20 I I, https://blog. oup.com/20 I I/02/quantum/.

16 Richard Conn Henry, “The Mental Universe," Nature, 436:29 (2005), https://doi.org/ I0.1 038/436029a. 